\title{
Alterstice
}

Revue internationale de la recherche interculturelle

International Journal of Intercultural Research

Revista International de la Investigacion Intercultural

\section{Soins et altérité : analyse comparée des regards et des pratiques des soignants}

\section{Marguerite Cognet et Sandra Bascougnano}

Volume 2, numéro 2, 2012

Regards pluriels sur les interventions sociales et de santé en contexte de diversité

URI : https://id.erudit.org/iderudit/1077561ar

DOI : https://doi.org/10.7202/1077561ar

Aller au sommaire du numéro

Éditeur(s)

Alterstice

ISSN

1923-919X (numérique)

Découvrir la revue

Citer cet article

Cognet, M. \& Bascougnano, S. (2012). Soins et altérité : analyse comparée des regards et des pratiques des soignants. Alterstice, 2(2), 9-21.

https://doi.org/10.7202/1077561ar

\section{Résumé de l'article}

L'analyse comparée des regards et des pratiques des soignants en France et au Québec permet d'interroger les idéaux portés par les modèles politiques d'intégration des populations immigrées et dits " de gestion de la diversité ». À partir de travaux empiriques menés à Montréal, Paris et Saint-Denis de la Réunion, nous montrons que les regards que les professionnels de la santé portent sur les populations altérisées - ethnicisées ou racisées - comme les pratiques qu'ils déploient à leur endroit restituent des tensions historiques mal contenues par les idéologies politiques. Les regards et les pratiques soignantes sont autant de révélateurs de figures d'altérité historiquement construites visant toujours à maintenir alter pour mieux préserver ego. Ce constat met en évidence le fait suivant : que la différence soit niée (modèle républicain) ou qu'elle soit mise en avant (modèle multiculturaliste), elle reste celle des autres. Autrement dit, la relation majoritaire/minoritaires reste impensée, seule la différence supposée des minoritaires justifie le questionnement des limites du modèle de l'intégration à la française ou de celles du multiculturalisme canadien. 


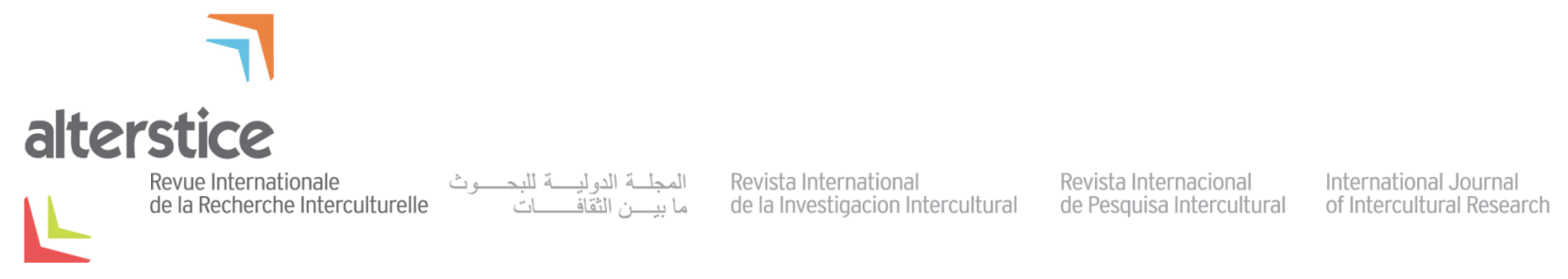

ARTICLE THÉMATIQUE

\section{Soins et altérité : analyse comparée des regards et des pratiques des soignants}

Marguerite Cognet $^{1}$ et Sandra Bascougnano ${ }^{2}$

\section{Résumé}

L'analyse comparée des regards et des pratiques des soignants en France et au Québec permet d'interroger les idéaux portés par les modèles politiques d’intégration des populations immigrées et dits « de gestion de la diversité ". À partir de travaux empiriques menés à Montréal, Paris et Saint-Denis de la Réunion, nous montrons que les regards que les professionnels de la santé portent sur les populations altérisées - ethnicisées ou racisées comme les pratiques qu'ils déploient à leur endroit restituent des tensions historiques mal contenues par les idéologies politiques. Les regards et les pratiques soignantes sont autant de révélateurs de figures d’altérité historiquement construites visant toujours à maintenir alter pour mieux préserver ego. Ce constat met en évidence le fait suivant: que la différence soit niée (modèle républicain) ou qu'elle soit mise en avant (modèle multiculturaliste), elle reste celle des autres. Autrement dit, la relation majoritaire/minoritaires reste impensée, seule la différence supposée des minoritaires justifie le questionnement des limites du modèle de l'intégration à la française ou de celles du multiculturalisme canadien.

\section{Rattachement des auteures}

${ }^{1}$ Université Paris Diderot et UMR 7032 URMIS - SOLIIS, Paris, France; ${ }^{2}$ Université de Bretagne occidentale et Atelier de recherche sociologique (ARS), France

\section{Correspondance}

marguerite.cognet@univ-paris-diderot.fr

\section{Mots clés}

pratique professionnelle; institution de la santé; ethnicisation; politique d'intégration

\section{Pour citer cet article :}

Cognet, M. et Bascougnano, S. (2012). Soins et altérité : analyse comparée des regards et des pratiques des soignants. Alterstice, 2(2), 9-22. 


\section{Introduction}

Cet article a pour objectif l'analyse comparée des regards et des pratiques des professionnels de la santé et des services sociaux en lien avec les idéaux portés en France et au Canada/Québec par les modèles politiques d'intégration des populations immigrées et dits " de gestion " des minorités ethno-raciales ${ }^{1}$ encore catégorisées comme autant de "communautés culturelles». Dans l'un et l'autre des deux pays, les questions d'altérité en lien avec l'installation des populations migrantes et de leurs descendances - subsumées sous le qualificatif singulier de "populations issues de l'immigration » - font l'objet de débats et de tensions (tensions d'ailleurs mises sous les feux de l'actualité au Québec lors du débat sur les accommodements raisonnables qui a accompagné les travaux de la Commission Bouchard-Taylor ${ }^{2}$ ).

Le Canada revendique son attachement au multiculturalisme, le Québec y adhèrant en partie tout en défendant le fait français par l'adoption d'un modèle alternatif dit de l'interculturalisme (Winter, 2006). La spécificité étant alors, dans le cadre d'un modèle pluraliste, de promouvoir l'intégration des différentes traditions culturelles en vue d'éviter des risques de marginalisation et de fragmentation du tissu social (Bouchard, 2012). La France, de son côté, reste attachée au modèle républicain et mise toujours sur l'universalité des droits et le principe d'égalité du citoyen de l'État-nation. II demeure que dans l'une et l'autre de ces sociétés, des faits et des pratiques politiques ont remis - et remettent - en cause ces modèles dans leurs applications. Le Canada, comme le Québec, se recentre sur la citoyenneté, voire sur une certaine canadianité (Labelle, 2005; Labelle et Salée, 1999). En France, on observe des politiques et des pratiques de gestion différenciées entre la métropole et les territoires hérités de la colonisation ainsi que l'émergence, dans l'hexagone, d'une critique du modèle républicain traditionnel (Streiff-Fénart, 2002; Wieviorka, 1999; Wiewiorka et Ohana, 2001). L'histoire passée et présente des pratiques sociales confronte les idéologies politiques, pose la question de leurs limites et nous paraît mettre en exergue des tensions entre idéologies et intérêts, entre une certaine idée sociale et politique de la démocratie et les rationalités économiques des logiques néolibérales.

Nous voulons discuter ici de ces modèles politiques et des tensions qui les accompagnent. Après un bref rappel des contextes historiques dans lesquels se sont édifiées et diffusées de grandes figures d'altérité, nous reprendrons les résultats de plusieurs études empiriques menées à Montréal, à Paris et à Saint-Denis de la Réunion (Bascougnano, 2009; Cognet, Bascougnano et Adam-Vezina, 2009; Cognet, Bertot, Couturier, Rhéaume et Fournier, 2005; Cognet, Couturier, Rhéaume et Bertot, 2010; Saillant, Chateauneuf, Cognet et Charland, 2008; Saillant et collab., 2006) pour explorer les regards que portent aujourd'hui les professionnels de la santé sur les populations «issues de l'immigration » - minorités ethnicisées ou racisées - et les pratiques qu'ils déploient à leur endroit du fait de ces regards. Nous reviendrons en conclusion sur les modèles politiques canadiens et français et nous essaierons de montrer en quoi ces regards soignants portent en eux-mêmes les tensions, voire les contradictions, qui soustendent les besoins de main-d'œuvre et le maintien d'une position hégémonique des pays du Nord sur les pays du Sud.

\section{Les figures de l'autre : histoire passée et présente de la barbarisation}

De la conquête du Nouveau-Monde au XV ${ }^{e}$ siècle à l'expansion coloniale accélérée au milieu du XIX ${ }^{e}$; du premier voyage de Jacques Cartier (1534) et de la constitution de la Nouvelle-France à sa cession aux Anglais en 1763 et à l'émergence de la Grande-Bretagne comme $1^{\text {re }}$ puissance coloniale du monde, puis à celle de l'empire colonial de la III République française jusqu'à la Seconde Guerre mondiale, les rapports de domination se sont historiquement

\footnotetext{
${ }^{1}$ Nous utilisons ce terme à dessein dans la mesure où il nous paraît restituer la construction d'une certaine altérité à partir de traits physiques et/ou culturels sélectionnés à l'aune des rapports de force qui ont accompagné et accompagnent les rapports entre les groupes sociaux intra- et inter-nationaux (voir De Rudder, Poiret et Vourc'h, 2000)

2 Les crispations sociales relatives aux accommodements raisonnables accentuées un peu partout au Canada depuis le retentissement de l'attentat aux États-Unis du 11 septembre 2001 ont poussé le gouvernement du Québec à créer une Commission de consultation sur les pratiques d'accommodement reliées aux différences culturelles, commission plus connue sous le nom de ses responsables, Messieurs Bouchard et Taylor. Le rapport publié au mois de mai 2008 a avivé le débat social sur ces enjeux sociétaux.
} 
établis sur des processus d'altérisation et de hiérarchisation ${ }^{3}$ des populations conquises au moyen d'un mécanisme principal qu'à l'instar de Taguieff (1998) nous appellerons la barbarisation ${ }^{4}$ : la dépréciation des qualités de ces populations conquises (valeurs, croyances, pratiques, normes) au profit de la survalorisation de celles des conquérants, ainsi que l'essentialisation de ces qualités.

Sous les empires coloniaux britanniques et français, dont le Canada fait alors partie intégrante, ont ainsi été construites au gré des conquêtes des représentations de l'autre à l'aune des besoins des colonisateurs. Aux côtés des grandes figures du juif et du mahométan qui émergent en Occident au milieu du xv siècle, et à partir desquelles se fondent les unités politiques des États européens (les ennemis de l'intérieur), vont s'édifier des figures d'altérité sur les nouveaux territoires conquis: l'indien autochtone d'Amérique, médiocre main-d'œuvre, qui, quand il n'est pas cannibale, ne peut-être qu'un éternel mineur incapable d'assumer son devenir; la figure du noir, descendant de Cham et doté de caractéristiques qui en font un esclave par "nature "; viendra ensuite l'indigène qui partage avec le sauvage des caractéristiques des « races inférieures ». Ces grandes figures n'excluent pas des figures plus locales comme celle des Panis, esclaves amérindiens au Québec (Trudel, 2004). Dans le même temps s'est construite une représentation idéalisée du colonisateur (Memmi, 2005).

Les violences réelles et symboliques qui accompagneront la conquête et la colonisation trouveront leur exutoire dans la mise en forme d'un ordre hiérarchisé racisé qui classera les Blancs au sommet, les Noirs au plus bas et, entre eux, tous les autres colonisés (Bouamama et Tevanian, 2006).

Ces figures ont donné sens à la conquête de l'autre, elles ont fondé la légitimité de l'empire colonial sous le label de la mission civilisatrice. Inévitablement, ces figures ont « irrigué la pensée occidentale » (Blanchard et Bancel, 1998, p. 13) et constitué le fond mythique à partir duquel sont pensés le rapport à l'autre et le rapport à soi. Le soi devenant la norme ethnocentrée, l'autre son négatif.

Stéréotypées, ces figures ont été diffusées à tous les niveaux des sociétés coloniales via tous les organes de communication - de la presse aux manuels scolaires en passant par les cartes postales et les expositions coloniales. Elles se sont incrustées dans les imaginaires (Blanchard et Bancel, 1998, p. 13). La décolonisation des territoires et la reconfiguration des rapports internationaux postcoloniaux n'ont pas fait table rase de ces figures qui, si elles peuvent évoluer dans le contenu qui leur est associé ${ }^{5}$, constituent des ressources cognitives, des manières de penser et d'interpréter le monde, une boîte à outils d'assignations identitaires normatives ${ }^{6}$, toujours mobilisables dans le cours des interactions pour orienter les conduites. Et si elles ont acquis une certaine pérennité dans l'espace social, il n'y a pas de raison de croire qu'elles ne pénètrent pas tous les systèmes y compris celui de la santé. D'autant que la construction et la diffusion de ces figures d'altérité ne furent ce qu'elles furent que parce qu'elles ont été confortées par une autorité scientifique, les allégations des sciences naissantes de l’homme et des sciences médicales.

\footnotetext{
${ }^{3}$ Si la mise en altérité est antérieure à la conquête comme il est possible de le voir dans les carnets de Christophe Colomb, où chez lui comme d'autres les premières réactions qu'occasionnent la découverte de l'Amérique et de son peuple sont marquées par l'exaltation de ce qu'il considère être l'ingénuité et la beauté primitives (Todorov, 1991), la conquête marque un autre temps de ce procès d'altérisation où l'autre est désormais catégorisé et prend place dans un rang hiérarchique.

${ }^{4}$ L'auteur enregistre trois opérations au cœur de la pensée raciste : l'essentialisme, qui consiste à réduire l'individu au statut de représentant quelconque de son groupe d'appartenance, érigé en nature ou en essence, fixe et insurmontable (naître tel, c'est être tel); la stigmatisation, qui vise à exclure symboliquement (voire réellement) les individus ainsi catégorisés au moyen d'une manipulation de stéréotypes négatifs et enfin la barbarisation qui est la déshumanisation partielle ou totale des catégories ainsi créées.

${ }^{5}$ Les représentations sociales que constituent les stéréotypes et les préjugés qui les accompagnent conservent le plus souvent un noyau dur (ou central) qui se caractérise par sa stabilité et sa cohérence. Il résiste aux changements et assure la permanence de la représentation (Bauer, 2011). Nous pouvons ici considérer que ce noyau est fondé autour de la hiérarchisation des supposées "races" ou " ethnies" indépendamment de la modification à la marge de certains éléments. Par exemple, l'idée que les juifs pourraient se reconnaître à un nez "crochu », stéréotype très présent dans les caricatures jusque dans les années 1940, est aujourd'hui atténuée au profit de marques (traits) attitudinales plus adéquates à la rectitude politique contemporaine.

${ }^{6}$ Abric (1994, p. 17) souligne que les représentations sociales ont une fonction normative : la représentation sociale « définit ce qui est licite, tolérable ou inacceptable dans un contexte social donné ».
} 


\section{Le rôle de la médecine comme science de l'autre}

Il est sans doute opportun de rappeler ce rôle. La médecine fut un des vecteurs majeurs de la mission civilisatrice dont se parait l'entreprise coloniale. L'accent est mis sur la vulnérabilité du monde primitif, vulnérabilité que la colonisation et la modernisation ne font qu'accroître et qui le pousse à se réfugier dans ses traditions. Le colonisé est de toute évidence mal préparé à lutter contre les maladies et paraît soumis aux pulsions les plus violentes (Carothers, 1953). Seuls la civilisation, les bienfaits de la médecine et l'évangélisation peuvent le soustraire à ces mauvaises influences. Ce qui, dans les discours médicaux, vaut pour "l'Africain » vaut tout autant pour l'Indien d'Amérique (Roy, 2004). Et si les médecins, examinent, marquent, classent microbes et populations (Dozon, 1985), il s'agit aussi d'analyser et de classer les traits raciaux ou culturels propres à expliquer la personnalité des indigènes, et bien sûr de trouver les marqueurs de leur animosité et de leur violence à l'égard des colons.

Cette rhétorique de la différence de l'autre par nature ou par culture n'est pas davantage absente de la médecine occidentale contemporaine, quand bien même elle prend des formes différentes ou plus édulcorées (Fassin, 1997, 2000). Elle est sensiblement perceptible dans les milieux de santé quand les praticiens se sentent démunis face aux échecs des interventions auprès de la population immigrante "qu'est-ce que vous voulez faire avec eux [les "Africains »], ils viennent, ils ne viennent pas, ils arrivent quand ils veulent... ils consultent à l'africaine !" (médecin, CHU parisien). Elle peut devenir patente à différents moments des trajectoires thérapeutiques:

- Dans la prévention, où elle peut être instrumentalisée par la notion de risque, notion dont les contours ne sont pas simples, avec la stigmatisation de "groupes à risque " et la tentative de correction par la notion de " comportements à risque » et avec laquelle on se demande toujours s'il est question de la santé de l'individu ou du corps social qui le côtoie, ou des deux mais, pour parler en termes plus foucaldiens, dans une perspective de biopouvoir (Fassin, 2001a, 2001b, 2005; Foucault, 1976).

- Dans le cours des traitements thérapeutiques, avec le recours à la notion d'observance renvoyant toujours l'individu à une certaine incapacité psychologique ou culturelle à suivre son traitement, à suivre les prescriptions normatives, à limiter les risques de développement de résistances des virus et des bactéries, bref à prendre ses responsabilités de citoyen patient-sentinelle (Cognet, Adam-Vézina et Braud, 2010; Pinell, 1992).

Ces notions de risque et d'observance nous apparaissent de fait, dans les soins auprès de personnes vues comme différentes, comme des outils de gestion de "leur» différence ainsi que vont le montrer les exemples de personnes séropositives au VIH-sida.

\section{Figures d'altérité en France}

La lecture des figures d'altérité en France proposée ici s'appuie sur une recherche menée entre 2003 et 2009 (Bascougnano, 2009). Un premier terrain d'enquête, fait d'observations in situ et d'entretiens semi-directifs avec les soignants, a été investigué au sein de deux services hospitaliers réunionnais où sont prises en charge des personnes séropositives au VIH entre août 2003 et fin janvier 2004. Un second terrain a ensuite été réalisé principalement dans un service hospitalier parisien de même nature entre avril et juillet 2005. Là encore les données ont été collectées par observations directes des interactions soignants-soignés et soignants-soignants (réunions, pauses, etc.) dans un service spécialisé dans les prises en charge de patients séropositifs. La période d'observation a été complétée par des entretiens semi-directifs auprès des soignants. Ces deux études ont constitué les matériaux empiriques d'une thèse doctorale (Bascougnano, 2009) Les données recueillies ont ensuite été enrichies à l'occasion d'une recherche collective commanditée par la DREES-MiRe, "Traitement différentiel dans les parcours thérapeutiques. Maladies infectieuses: VIH-sida, tuberculose ", réalisée sous la direction de Cognet de 2006 à 2008 (Cognet, Bascougnano, Adam-Vezina, 2009). Cette étude a été menée dans trois Centres hospitaliers universitaires (CHU) d'île-de-France au sein de services spécialisés en maladies infectieuses et immunologie. La méthodologie déployée combinait cette fois des recueils de données objectives (données cliniques et thérapeutiques des dossiers patients) et subjectives auprès d'une centaine de patients (questionnaires ouverts). Les 17 médecins référents des patients impliqués ont répondu à un entretien de rétroaction (Clôt, 1999; Theureau, 2010) les invitant à commenter et expliciter leurs orientations et décisions thérapeutiques.

Alterstice - Revue Internationale de la Recherche Interculturelle, vol. 2, $n^{\circ} 2$ 


\section{Les "Réunionnais précaires"}

La Réunion est un département français d'outre-mer, situé à 10000 km de la France métropolitaine. II s'agit d'une ancienne colonie française devenue un Département français d'outre-mer (DOM) en 1946. La Réunion était inhabitée quand la France s'est approprié l'île, au XVII siècle. Les colons ont rapidement recouru à une maind'œuvre d'esclaves originaires d'Afrique de l'Est et de Madagascar. En 1848, à la suite de l'abolition de l'esclavage, ils ont alors fait appel à une main d'œuvre dite d'engagés, essentiellement des Indiens originaires du sud de l'Inde. D’autres migrations se sont ensuite succédé jusqu'à aujourd'hui : des Indiens musulmans du nord de l'Inde, des Chinois et à présent des Français métropolitains, des Comoriens et des Mahorais.

De migrations en migrations, l'île de la Réunion a vu sa population croître notablement. Elle dépasse aujourd’hui les 700000 habitants. Société plurielle, sublimée par certains intellectuels et artistes réunionnais qui voient en elle la préfiguration de l'avenir de la planète, convaincus ou presque de l'absence de racisme en cet espace où tous vivraient ensemble harmonieusement et dont le métissage se voudrait le symbole de cette réussite, la Réunion s'est en réalité peuplée, pour une large part, durant les périodes esclavagiste et colonialiste. Les contextes historiques de ces migrations ont construit des relations interethniques prises dans des rapports de domination qui continuent, sous des formes différentes, d'inférer sur la vie sociale réunionnaise. Les rapports de domination ne sont certes plus du type maître/esclave et maître/engagé, mais à chaque groupe ethnicisé sont associés des traits physiques, psychiques et comportementaux qui tendent à être essentialisés et participent de la structuration de la vie sociale réunionnaise.

Si le détour par le passé s'avère nécessaire pour comprendre les relations interethniques à la Réunion aujourd'hui, le sens de ces relations consiste bien en une réappropriation et une réinterprétation de cette histoire au présent. Ce sont les rapports de domination qui se maintiennent plus que des stéréotypes. Les traits stéréotypés ne sont plus nécessairement aujourd’hui les mêmes que ceux usités hier,

l'histoire étant utilisée par les acteurs comme stock, comme réservoir d'images, dans des dynamiques sociales et politiques qu'il faut appréhender au présent [...]. Au lieu de l'histoire coloniale, c'est alors la mémoire coloniale que l'on va peut-être trouver comme un facteur explicatif du stéréotypage contemporain de l'immigration - la mémoire, c'est-à-dire une construction au présent, à la fois sociale et subjective, malléable aussi, du sens du passé. (Lorcerie, 1997, p. 78-79)

De fait, de nouveaux traits comportementaux ont émergé qui viennent réaffirmer l'existence sociale de groupes ethniques, renforcer les frontières, repenser les différences et donc les inégalités. Ainsi, les « Cafres » d'aujourd'hui sont considérés comme les descendants des esclaves asservis à leurs maîtres. Aujourd'hui, ils sont pensés comme étant dans l'incapacité de se prendre eux-mêmes en charge face à l'absence d'une autorité et formeraient une population d'assistés, survivant grâce à "l'argent braguette » - expression couramment employée à la Réunion pour parler des allocations familiales. Certains imaginaires, construits du temps où la Réunion était une société esclavagiste, sont parfois réinvestis aujourd'hui, tel celui d'une " sexualité noire ». D'autres émergent, participant au maintien de la différenciation et de la hiérarchisation des groupes ethnicisés qui coexistent à la Réunion. Lorcerie (1997) a choisi de parler de "stéréotypages" afin de rendre compte du caractère processuel de la construction de ces représentations sociales qui visent à maintenir ou à faire tenir des rapports de domination. Si le " métissage » reste largement sollicité dans les discours des jeunes réunionnais d'aujourd'hui, les revendications actuelles de reconnaissance d'un passé esclavagiste et les quelques manifestations d'hostilité à l'égard de métropolitains témoignent de tensions réelles à la Réunion. Les patients métropolitains rencontrés à la Réunion et ayant également vécu en Guadeloupe ou en Martinique mettent en avant le moindre racisme perçu à la Réunion par rapport à ce qu'ils ont connu dans ces deux autres îles françaises. Les inégalités restent fortement ancrées sans nécessairement, cependant, être ostensiblement affichées.

Au sein des services hospitaliers étudiés, certains malades sont qualifiés par les soignants de « Réunionnais précaires ". Ces patients sont présentés comme posant des problèmes ou, à tout le moins, comme étant fortement susceptibles d'en poser dans le cours de leur prise en charge et en dehors d'elle. En effet, à ce groupe est associé un ensemble de caractéristiques comportementales et psychiques jugées néfastes à une bonne observance thérapeutique, d'une part, et susceptibles d'engendrer des prises de risques inconsidérés (rapports sexuels non protégés), d'autre part. 
Dès lors, les soignants ont opté pour une prise en charge spécifique, visant à favoriser une bonne observance et à limiter ces «comportements à risques". Ce traitement particulier appliqué aux "Réunionnais précaires » repose principalement sur de la surveillance et du contrôle, telle que la prise de traitements en observation directe.

Au final, l'adéquation se traduit en équation où les caractéristiques s'additionnent (origine réunionnaise + précarité + comportements déviants [alcool, cannabis, violence] + problèmes psychiatriques + assistanat social) pour créer une catégorie d'individus stigmatisés, dont il faut assurer la surveillance et qu'il faut endiguer sur le plan sociosanitaire. Or toutes nos observations concourent à prouver que les individus qui se trouvent ordinairement décrits sous ces traits constituent un groupe ethnicisé (assigné dans une différence culturelle irréductible) bien repéré à la Réunion puisqu'il s'agit des individus constitués en un groupe historiquement exclu de la citoyenneté ordinaire, des individus issus des fractions sociales les plus dominées d'un ordre racisé (organisation sociétale entre des groupes racisés, assignés dans une différence de nature transgénérationnelle) et raciste qui prévalait officiellement dans l’État colonial : les « Cafres », les descendants des esclaves africains et malgaches.

Les " Africains » à Paris

Qu'en est-il sur le territoire métropolitain de la France? Le discours égalitaire du citoyen par la République et la négation des groupes ethniques génèrent-ils des regards et des comportements différents à l'égard des populations minorisées?

Au sein des services hospitaliers d'île-de-France étudiés, les patients originaires des anciennes colonies françaises d'Afrique sub-saharienne sont nombreux. Ces patients, et avec eux des malades originaires d'autres pays d'Afrique sub-saharienne, sont communément appelés par les soignants - hors de la présence des patients - les "Africains », même si les praticiens apportent fréquemment une précision sur la nationalité du patient dont il souhaite parler à ses confrères. Ces "Africains " n'échappent pas à la résurgence de ces imaginaires coloniaux et aux rapports de domination qui y sont associés. Comme dans le contexte réunionnais, ce groupe fait l'objet d'une (re)construction autour des notions d'observance et de risque sur la base d'un ensemble d'arguments relatifs à leur incapacité (du fait de leur précarité socio-économique et juridique et de leur culture), et à la dangerosité de leurs comportements sexuels (machisme, polygamie, refus d'usage du préservatif, etc.).

Comme à l'égard des "Réunionnais précaires ", les "Africains » font l'objet d'un traitement différentiel, mais qui prend une autre forme puisqu'il s'agit d'amener ces patients vers l'observance en les éduquant. Autrement dit, s'ils sont incapables, cette incapacité n'est que temporaire. Autrement dit, ils peuvent apprendre et devenir de « bons patients ". Les différences (toujours négatives) imputées à ce groupe sont donc moins essentialisées puisqu'il demeure possible que ces patients changent. Néanmoins, des pratiques de contrôle et de surveillance peuvent intervenir au besoin.

II semble que le poids du discours républicain se fasse plus prégnant en France métropolitaine, générant préférentiellement des pratiques d'éducation plutôt que des pratiques de surveillance. Les mécanismes de (re)construction de l'Autre demeurent en revanche les mêmes, mettant en scène, pour les "Réunionnais précaires ", la figure du sauvage inéducable et, pour les "Africains » de Paris, celle de l'indigène évolué. Au nom d'arguments d'ordre médical et de santé publique s'ensuivent des traitements différentiels qui se veulent, d'un point de vue médical, bénéfiques puisque pensés mieux adaptés à ces groupes.

\section{Les figures de l'autre au Québec}

Au cours de recherches que nous avons conduites au Québec dans les milieux de la santé et des services sociaux, par la réalisation d'entretiens auprès de praticiens (infirmières, travailleurs sociaux et aides familiaux et sociaux), nous avons recueilli un certain nombre de ces figures de l'autre produites par les professionnels œuvrant dans des structures de services de première ligne, que ce soit en centres locaux de services communautaires (CLSC, aujourd'hui centre de santé et services sociaux, CSSS), dans des associations ou pour le compte d'agences privées de Montréal et de plusieurs autres villes du Québec (Québec, Sherbrooke, Trois-Rivières). Nous avons conduit des entrevues semi-structurées auprès de ces différents groupes de soignantes et soignants et auprès de leurs employeurs ou supérieurs hiérarchiques (Cognet, 2002, 2003, 2004a, 2004b, 2004c; Cognet et collab., 2005; Cognet, Bourgon, Bouvier et Dufour, 2006; Cognet et collab., 2010; Cognet et Saillant, 2003). Certaines de ces 
figures puisent dans les imaginaires coloniaux tels qu'ils ont été construits au cours du colonialisme interne et au fur et à mesure des vagues qui ont fait l'histoire du Canada (Calliste, 1993; Ng, 1993; Roy, 2003 et 2004 ) et comme en France ont persisté au travers de l'histoire, d'autres semblent construites dans l'expérience de l'intervention et peuvent apparaître davantage sous l'influence du multiculturalisme ou de l'interculturalisme. A priori, dans ces milieux, les immigrants bénéficient d'un préjugé positif. La $1^{\text {re }}$ figure évoquée est celle du combattant. L'immigrant est l'homme qui a eu le courage de partir de son pays pour l'inconnu, de quitter ses repères et ses proches pour se mettre en quête d'un autre avenir : « ils n'ont pas choisi d'être ici et c'est difficile de s'intégrer, puis ils sont partis des fois sans saluer leur famille et ici ils arrivent comme réfugié. Ils ne peuvent plus vivre, leurs parents, leur famille c'est épouvantable » (infirmière). C'est ici une figure valorisée, celle de l'homme et/ou du père qui affronte l'extérieur pour faire vivre les siens.

La figure exacerbée du combattant est celle du survivant, que les praticiens évoquent souvent quand ils parlent des réfugiés. À la fois fort et vulnérable, le demandeur d'asile ou le réfugié bénéficie d'une représentation qui force l'admiration. Il y est montré justement d'autant plus fort, d'autant plus courageux qu'on se le représente comme survivant des conditions de vie et de traitements inhumains :

Quand je pars de là, euh, je ne peux pas m'attribuer ce qu'a vécu la personne... c'est sûr que quand j'entends dire qu'une personne a été tor... torturée, là, ça... ça me fait... ça... ça me fait vraiment quelque chose puis... moi je pense que j'arrive à travailler avec toutes ces émotions là parce que... en me... en me rebranchant sur les forces de la personne. (travailleuse sociale)

L'immigrant est ici celui qui vit une période de transformation, de transition entre sa vie d'avant et sa nouvelle vie et qui connaît une perte de ses repères et références. Ce sont les conséquences de cette situation qui l'amène à consulter.

Notre travail ça va souvent loin. L'année passée, j'ai aidé deux petites madames à l'accouchement. Comme il n'y avait pas de mari qui allait à l'accouchement, j'ai joué un petit peu le rôle de la ma tante puis je suis allée pendant l'accouchement. La première qui avait accouché, le médecin m'a demandé si je voulais couper le cordon, pourtant j'en ai eu des enfants, mais là des grosses larmes me coulaient. Elle était comme ça de prise et elle a dit à son mari : " j'ai fait pleurer Jeanne ". J'ai dû lui expliquer qu'on peut pleurer de joie. Et là le cœur il m'a fait comme ça : boum ! » (travailleuse sociale)

Auprès de cette figure, les praticiens se voient investis d'une signifiance particulière pour accompagner le nouvel arrivant dans cette errance entre deux mondes :

II m'est arrivé et il m'arrive encore de pleurer en présence de réfugiés quand ils me racontent leur histoire. De les prendre dans mes bras, puis de pleurer. Puis c'est comme... rien que ça que je suis capable de leur offrir là. Et à un moment donné, tu ne sais plus qui berce qui... "; "Mais ça, je trouve que c'est un privilège. C'est un cadeau. Moi quand les gens ils me font... ont cette confiance-là, je trouve que c'est un cadeau. Ah oui. Moi, moi c'est vraiment... C'est pas lourd pour moi. C'est pas quelque chose que je porte en lourdeur. C'est quelque chose que je porte en connaissances puis en... Ca me donne l'énergie pour... pour faire ce que je fais finalement. Tu sais, c'est tout ça mélangé. Tu sais, c'est difficile à identifier. Mais, tu sais, d'être dépositaire de ça quand les, les... Mon Rwandais me racontait après trois ans, après trois ans que je le fréquentais, comment il a vécu au Rwanda des choses. Ah! C'était... Ah! (responsable association)

Mais d'autres figures surgissent dans le cours des interventions.

Une $2^{\mathrm{e}}$ figure apparaît dans les discours, celle du discriminé, en raison de son origine ethnique ou de son assignation dans un groupe racialisé. Dans leurs interventions, les praticiens et praticiennes s'efforcent alors de considérer les obstacles structurels que rencontrent ces clients particuliers.

Quand il a eu une difficulté soit avec les euh... une défection de travail, une question de discrimination, ah! une question de par exemple les criminels, euh... toutes ces affaires-là. Alors, ça une question juridique, c'est un peu la même chose. Une question de femmes aussi. On touche tout ce que, concernant la violence conjugale. La pauvreté des femmes. La monoparentalité. Toutes les affaires qui concernent des femmes un peu... qui sont défavorisées. (travailleur social)

C'est une figure qu'il faut accompagner dans ses difficultés mais qu'il faut également tenter d'armer (empowerer) contre ces obstacles qui, du point de vue des praticiens, resteront sans doute longtemps sur son chemin : « il y a FE, c'est un bloc discussion où les femmes cherchent des outils pour s'entraider comme... pour lutter contre la discrimination. " (infirmière). 
Ces figures construites fonctionnent comme des figures attendues dans la relation clinique. Elles présentent une face positive de l'autre comme de soi et projettent ainsi des sortes d'idéaux-types construits à la fois par les cadres sociaux et politiques et en partie confortés par l'expérience d'intervention. Mais comme toutes figures attendues, quand l'autre, pour une raison ou une autre, n'y correspond pas, ce sont alors des faces plus négatives qui surgissent. Il en va ainsi de la figure du traditionnaliste.

Au point de vue haïtien ils sont encore beaucoup dans le... dans le traditionnel et ce sont des gens qui sont difficiles à atteindre parce que je pense qu'ils ont encore cette crainte du... de la dénonciation, de... je crois que ce sont des gens qui sont marqués dans leurs gènes sur... sur tout ce qu'ils ont pu vivre en Haïti, et puis il y a encore, même ici à Montréal on sait qu'il y a encore des séances de vaudou, de choses comme ça, là qui sont... sur lesquelles ils expliquent plein de choses. (infirmière)

... des gens d'origine musulmane, souvent comme Marocains ou des gens d'origine d'arabe, des fois il faut qu'on s'adapte que il faut être avec toi-même, c'est la femme qui vient consulter, il faut qu'on s'adapte que le mari soit présent parce que le mari veut tout le temps entrer là, pis des fois pour nous c'est comme une contrainte, mais des fois il faut accepter ça, pis souvent c'est le mari qui répond à la place de la femme, mais en tout cas c'est une façon de... peut-être c'est une façon que nous... certaines façons si on a jamais pas vécu dans ces cultures-là on comprend pas pourquoi c'est comme ça mais c'est la façon qu'eux autres ils s'organisent! (infirmière)

Présentée ici moins comme des individus que membres de sociétés solidaires de type communautaire attachés à la tradition, cette figure est souvent opposée à celle du citoyen de la société d'accueil, figure de la modernité moins centrée sur les valeurs de la communauté, de la famille, et ayant depuis longtemps rompu avec la tradition. Mais cette figure a deux faces. Sous sa face positive, elle est élevée au rang d'exemple de société, celle du rêve perdu, alors que, sous sa face négative, elle est ramenée au rang d'une société archaïque qu'il faut finalement adapter aux conditions de la société moderne.

Ils [les Haïtiens] ont un... ils ont un flegme qui est différent du nôtre, c'est des gens des pays chauds, c'est des gens... mais quand on le comprend, moi je pense que les enfants vont être plus rapides que leurs parents parce qu'ils sont nés ici dans un climat qui fait qu'il faut se battre l'hiver pour pas avoir froid, se battre dans le sens il faut... t'sais on réagit, on a... on est bâti par l'endroit où on vit, on est conditionné par les changements de température. Ces personnes-là, c'est vrai qu'elles sont plus lentes, mais pas toutes, pas toutes, il y a des parents qui sont très vifs, très... [...] Alors les conscientiser ça prend peut-être plus de temps mais ça vaut la peine quand on réussit à faire changer quelque chose dans la vie d'un enfant c'est une belle victoire là pis c'est pour là qu'on... c'est pour ça qu'on est là. » (infirmière)

L'immigrant est aussi celui qui ne sait pas, qui est vu comme culturellement différent. Une différence qui le rend à peu près ignorant (voire totalement) du fonctionnement de la société québécoise, de ses lois, de ses codes sociaux, de ses mœurs et de ses habitudes. L'accent sur la culture pèse fortement, c'est ici la figure du transplanté culturel. «II y a certaines cultures qui sont plus difficiles que d'autres. Euh... II y a des cultures avec lesquelles j'ai plus de misère, personnellement, je pense là que d'autres. " (travailleur social). Une figure plus ambiguë dans la mesure où, quand on évoque certaines de ses pratiques, de ses croyances ou de ses coutumes, la figure de l'indigène n'est pas toujours très loin, voire celle du sauvage ou du barbare, de celui qui n'est pas tout à fait aussi civilisé que soi, qui n'a pas eu la chance de connaître les apports de la démocratie. Le fossé culturel existant entre l'immigré et sa société d'accueil ne peut s'atténuer que grâce au travail des praticiens qui vont agir comme des éducateurs pour l'amener à se transformer:

Au départ ça va arriver, par rapport à comment porter le bébé, ou le coucher... Il y a des choses qu'ils ont toujours faites, et tu te dis : bon, pour l'instant ça va bien puis c'est de lui faire comprendre que là il y a d'autres choses maintenant qu'il faut mettre en place. (infirmière)

C'est un client qui est... qui est très loin culturellement de la... de la culture d'ici, à qui on explique les choses et qui n'arrive pas à les saisir. Ça, c'est très difficile aussi. Et un client qui fonctionne à sa manière. Voilà! On aimerait le voir fonctionner de telle manière pour que ça puisse être facile pour lui, mais qui a des attitudes en plus de son... de, de ses... de son handicap en tant qu'immigrant ici pour accéder au marché du travail. En plus il a des attitudes personnelles qui ne nous facilitent pas le travail. (travailleuse sociale) 


\section{Différences ou similitudes entre les deux pays?}

$S^{\prime}$ il est assez clair que la résurgence de ces dernières figures prend place dans une relation tendue, où l'autre déroge aux attentes et provoque ainsi une sorte de besoin de réajustement permettant de redonner du sens à la relation et au rôle de soi, il nous reste à saisir pourquoi ces figures d'altérité présentent un certain nombre de similitudes entre le Québec et la France sur son territoire métropolitain ou sur ses territoires d'outre-mer. II nous faut également comprendre pourquoi, dans les deux pays, en dépit des différences entre le modèle républicain et le multiculturalisme/interculturalisme, les types de figures d'altérité diffèrent finalement peu de celles qui ont été élaborées au cours de la colonisation.

Il apparaît que dans les deux pays des éléments convergent. Les années 1960-1970, qui suivent la décolonisation, se caractérisent dans les deux pays par des changements sociaux démographiques importants :

- Les pays dont sont originaires les migrants changent. Les origines européennes se tarissent et les nouveaux arrivants viennent de plus en plus nombreux des pays du Sud, et parmi eux de pays des anciens empires coloniaux de la France et de l'Angleterre.

- Relativement à l'arrivée de ces populations et à leur installation définitive sur le territoire - en France l'immigration est de moins et moins perçue comme transitoire, liée au travail - le sentiment de "diversité culturelle » s'accroît.

- La récession généralisée, consécutive à la crise économique qui éclate en 1973, se traduit par une rupture entre le capitalisme et la démocratie sociale qui avaient, au cours des Trente glorieuses, assuré la croissance et l'essor de l'État-providence. Les conséquences sociales et économiques de cette rupture favorisent des crispations socio-identitaires entre nationaux et immigrants.

- Dans le même temps, des revendications identitaires de minorités nationales pour la reconnaissance de leur culture (dont le patrimoine linguistique) aiguisent les clivages internes. Au Canada, une cristallisation a eu lieu sur la demande des Canadiens francophones, en France sur les demandes régionalistes des Bretons, des Corses ou des Basques.

Les politiques qui seront mises en œuvre, au cours de ces années post-coloniales, seront des tentatives de réponse à ces tensions. Elles visent à la fois a) à assurer une certaine circulation de la main-d'œuvre propre aux besoins économiques intérieurs et, au-delà, à pérenniser l'hégémonie gagnée dans la période coloniale et b) à maintenir la cohésion sociale du pays par une intégration suffisante pour désamorcer les tensions tout en favorisant, explicitement (modèle français) ou implicitement (modèle canadien), le maintien de la culture dominante.

Elles sont de deux ordres: les politiques migratoires d'une part, les politiques d'intégration de l'autre. Et nous voudrions souligner, en en rappelant les traits saillants, que celles de la France, du Canada et du Québec ne divergent pas forcément autant qu'il y paraît à première vue.

\section{Les politiques migratoires}

Les politiques migratoires que la France adoptent dans le milieu des années 1970 et les années suivantes pourraient se résumer par un accès de plus en plus contrôlé au territoire et à la nationalité : affirmation d'une immigration zéro en 1974 et la négociation accrue de dérogation de visa pour le marché du travail (dont le décret de 1975 liant l'obtention du visa à la situation de l'emploi, soit l'amorce d'une politique sélective inavouée); réforme du code de nationalité en 1973 qui sera poursuivie dans les décennies suivantes par des mesures de plus en plus contraignantes.

Au Canada, l'ouverture qu'avaient occasionnée les nouveaux règlements des conditions d'entrée déposés en 1962, en éliminant toute sélection fondée sur la race, la religion ou l'origine nationale, voit sa portée réduite dès 1967 avec l'instauration de critères de sélection selon un système de points d'appréciation des qualités des demandeurs en fonction des besoins de l'économie. À compter de 1973, les femmes immigrant sous le Programme des aides familiaux résidents (PAFR) n'auront désormais qu'un statut temporaire sur le sol canadien. La Loi sur l'immigration de 1976 stipule que la sélection des étrangers se fait en fonction de «leur capacité à réussir leur établissement 
économique au Canada. " Cette même loi soumet le droit d’immigrer aux critères de bonne santé. Le Québec, qui a une certaine autorité sur ses propres immigrants, suit le même type de règles de sélection. La montée de contestations et des revendications identitaires de la part des communautés, des minorités nationales et des populations autochtones, l'influence de mouvements fondamentalistes religieux et la formation d'identités et $d^{\prime}$ institutions transnationales ou supranationales font craindre une détérioration du sentiment d'appartenance nationale que les modifications apportées à la loi de 1976 jusqu'à la loi sur la citoyenneté canadienne de 98 chercheront à endiguer par des mesures telles que l'augmentation de la durée de résidence permanente et l'examen d'intégration.

\section{Les politiques d'intégration}

En matière de politique d'intégration, ou peut-être devrions nous plutôt dire de "régulation des rapports interethniques ", la France comme le Canada sont à la recherche de modèles politiques qui pourraient à la fois assurer la cohésion sociale et permettre l'expression d'une certaine diversité.

Au Canada, la réponse à ce dilemme fut l'adoption du multiculturalisme en 1971 comme politique officielle, politique enchâssée dans la Constitution en 1982 comme élément de la Charte canadienne des droits et libertés et finalement érigée en loi en 1988 (Loi sur le multiculturalisme canadien).

En France, l'attachement au modèle républicain et la résistance à l'intrusion de tiers entre l'individu et l'Etat occasionnent plus de turbulences. Si l'on a envisagé nuls droits collectifs à des groupes particuliers, la reconnaissance des difficultés d'application de ce modèle par l'échec de l'intégration des jeunes descendants des populations qui, hier encore, étaient colonisées, conduit, bon an, mal an, à revisiter le modèle et à avancer vers des propositions alternatives encore en débat (Schnapper, 2007; Weil, 2005; Wiewiorka et Ohana, 2001). Au Québec, l'interculturalisme se veut d'abord une alternative au multiculturalisme, que les Canadiens francophones, et en particulier les Québécois indépendantistes (souverainistes), ont pris comme une manœuvre politique du Canada anglophone en vue de désamorcer leur revendication identitaire. L'interculturalisme puise dans une double tradition. D'une part, il est une politique inspirée du modèle français (dans des faits tels que la francisation obligée des enfants d'immigrants, il propose bien un certain assimilationnisme) et, en affirmant le fait français comme culture dominante, il résiste aux revendications identitaires des minorités du Québec que le multiculturalisme pourrait laisser entrevoir. D'autre part, en reconnaissant des " communautés culturelles » et en invitant à ouvrir la négociation sur la base de droits culturels en vue d'accommodements raisonnables, il reprend à son compte une partie du projet multiculturaliste qui caractérise la politique des pays rattachés au Commonwealth.

Finalement que disent ces politiques aux praticiens? D’un côté, les politiques migratoires, qui suivent des lignes assez semblables dans les deux pays, contribuent à maintenir l'idée qu'il y a de "bons immigrants " - ceux qui peuvent, veulent se conformer au plus vite aux modèles attendus et pour qui il est loisible de projeter la figure du combattant, du résilient - et de "beaucoup moins bons » - ceux dont il est supposé un écart plus ou moins irréductible et sur qui se projette plus facilement la figure du traditionnaliste. Ces distinctions constituent un jeu manichéen dont les coloniaux avaient l'art, distinguant dès les premières heures les "bons sauvages " des " mauvais » et plus tard les « indigènes évolués » de la masse des colonisés. Elles participent ainsi à l'entretien des figures négatives, dépréciatives de l'autre. D'un autre côté, les politiques d'intégration, quoique nous puissions considérer qu'elles n'en sont pas au même état d'avancement, nous paraissent néanmoins aller dans le même sens. Elles invitent à la recherche d'un compromis entre soi et l'autre désormais à l'intérieur mais, de façon explicite ou implicite, le modèle républicain, l'interculturalisme et le multiculturalisme reconnaissent la primauté d'une culture dominante et autorisent de ce fait les nationaux à rappeler à l'ordre les derniers venus qui pourraient se faire trop rétifs à la transformation de leurs pratiques.

Ainsi ces politiques disent un certain nombre de choses du rapport à l'autre, par exemple en dessinant la figure de l'immigrant souhaité au travers de propositions. Ces politiques peuvent également diffuser des messages subliminaux, notamment pour promouvoir une culture dominante. Le milieu de la santé n'étant pas davantage que d'autres imperméable à ces messages, ces derniers influent alors sur les figures mises en scène dans les discours des praticiens sur ces autres et dans leurs interventions auprès d'eux. Historiquement, les politiques ont été

Alterstice - Revue Internationale de la Recherche Interculturelle, vol. 2, $n^{\circ} 2$ 
utilisées pour institutionnaliser des traitements différentiels. Dans la colonisation, des codes particuliers ont été édictés pour réglementer le rapport de l'autre à soi (en France, le Code Noir en 1685 et le Code de l'Indigénat en 1881; des politiques similaires furent instaurées par les autres puissances coloniales). De ce fait, elles ont toujours contribué à dessiner des figures d'altérité. Les politiques actuelles ne démentent pas cette règle. Les figures d'altérité ethnicisées mises en scène dans les milieux de santé opèrent comme des rappels normatifs révélateurs d'une conception politique de l'intégration de l'étranger pensée dans les limites de la préservation des groupes dominants et de leurs valeurs. II s'agit de maintenir l'autre différent à distance ou de le transformer en semblable. D'autres thèses pourraient sans doute permettre de poursuivre la discussion de cette hypothèse telles que la thèse de la "recolonisation" défendue par des théoriciens des études post-coloniales (Lazarus, 2006) où la politique

occidentale favorise une combinatoire entre une politique extérieure impérialiste via, entre autres, les programmes d'ajustement structurel, et un certain appel au chauvinisme sur le front intérieur (Schell, cité par Lazarus, 2006, p. 105-106). Un travail à poursuivre.

\section{Références bibliographiques}

Abric, J.-C. (1994). Pratiques sociales et représentations. Paris : Presses universitaires de France.

Bascougnano, S. (2009). Savoirs, idéologies et traitements différentiels discriminants dans les rapports soignants/soignés. La prise en charge hospitalière du VIH-sida à la Réunion et en Île-de-France. (Mémoire de maîtrise). Université Paris Diderot.

Bauer, A. (dir.). (2011). La criminalité en France. Synthèse du rapport de l'Observatoire national de la délinquance et des réponses pénales. Paris : Institut national des hautes études de la sécurité et de la justice (INHESJ).

Blanchard, P. et Bancel, N. (1998). De l'indigène à l'immigré. Paris : Gallimard.

Bouamama, S. et Tevanian, P. (2006). Un racisme post-colonial. Dans N. Bancel, P. Blanchard et S. Lemaire (dir.), Culture post-coloniale 1961-2006 (p. 243-257). Paris : Autrement.

Bouchard, G. (2012). L'interculturalisme. Un point de vue québécois. Montréal : Boréal.

Calliste, A. (1993). Women of "Exceptional Merit" : Immigration of Carribean Nurses of Canada. Canadian Journal of Women and Law, 6, 85-102.

Carothers, J. (1953). The African Mind in Health and Disease: A study on Ethnopsychiatry. Genève : OMS.

Clôt, Y. (1999). La fonction psychologique du travail. Paris : Presses universitaires de France.

Cognet, M. (2002). La santé des immigrés à l'aune des modèles politiques d'intégration des étrangers. Bastidiana, 39-40, 97-130.

Cognet, M. (2003). Politiques d'immigration canadiennes et femmes d'ailleurs dans les emplois dans le secteur des soins et des services à domicile. Dans F. Saillant et M. Boulianne (dir.), Transformations sociales, genre et santé. Perspectives critiques et comparatives (p. 183-205). Québec: Presses de l'université Laval/Paris : L'Harmattan.

Cognet, M. (2004a). L'expérience du genre, de la classe sociale et de l'ethnicité dans le quotidien de travail des auxiliaires familiaux. Quoi de neuf, 14(8), 1-4.

Cognet, M. (2004b). La vulnérabilité des immigrés : Analyse d'une construction sociale. Dans F. Saillant, M. Clément et C. Gaucher (dir.), Identités Vulnérabilité, Communautés (p. 155-188). Québec : Éditions Nota Bene.

Cognet, M. (2004c). Les représentations des praticiens vis-à-vis de l'ethnicité dans la rencontre clinique, L'immigration et l'intégration au cœur des débats : recherches, politiques et pratiques. Communication présentée à la $7^{\mathrm{e}}$ Conférence Nationale Metropolis, Montréal, Canada.

Cognet, M., Adam-Vézina, E., avec la collaboration de R. Braud (2010). L'observance : Analyse critique des savoirs (rapport de recherche). Paris : Ministère de la Santé.

Cognet, M., Bascougnano, S. et Adam-Vezina, E. (2009). Traitement différentiel dans les parcours thérapeutiques. Maladies infectieuses: VIH-sida, tuberculose (rapport de recherche). Paris : Ministère de la santé. 
Cognet, M., Bertot J., Couturier Y., Rhéaume J. et Fournier B. (2005). Organisation des soins infirmiers de santé primaire en milieu pluriethnique. (Premier volet du projet Pratiques infirmières en milieu pluriethnique. Entre modèles culturels et modèles professionnels). Montréal : Centre de recherche et de formation du CSSS Côtedes-Neiges, Métro et Parc-Extension.

Cognet, M., Bourgon, A., Bouvier, L. et Dufour, L. (2006). Citoyenneté et soins de santé aux immigrants : Les infirmières jouent-elles un rôle dans la construction de la citoyenneté des immigrants au Québec? Cahiers de METISS(1), 25-36.

Cognet, M., Couturier, Y., Rhéaume, J. et Bertot, J. (2010). Soins infirmiers en milieu pluriethnique : points de vue et pratiques dans les établissements publics et privés face à la discrimination et au racisme. Dans A. Battaglini (dir.), Les services sociaux et de santé en contexte pluriethnique (p. 251-276). Québec : Saint-Martin.

Cognet, M. et Saillant, F. (2003, 19-23 mai). Les représentations de I'humanitaire et du communautaire chez des intervenants auprès de réfugiés de 5 régions du Québec. Communication présentée au $71^{\mathrm{e}}$ Congrès de I'ACFAS - Savoirs partagés, Rimouski, Canada.

De Rudder, V., Poiret, C. et Vourc'h, F. (2000). L'inégalité raciste. L'universalité républicaine à l'épreuve. Paris : Presses universitaires de France.

Dozon, J. (1985). Quand les pastoriens traquaient la maladie du sommeil. Sciences sociales et santé, 3(3-4), 27-56.

Fassin, D. (1997). L'internationalisation de la santé entre culturalisme et universalisme. Esprit, 229, 83-105.

Fassin, D. (2000). Les politiques de l'ethnopsychiatrie. La psyché africaine, des colonies africaines aux banlieues parisiennes. L'Homme, 153, 231-250.

Fassin, D. (2001a). Au cœur de la cité salubre. Dans J.-P. Dozon et D. Fassin (dir.), Critique de la santé publique (p. 47-73). Paris : Balland.

Fassin, D. (2001b). Le culturalisme pratique de la santé publique. Critique d'un sens commun. Dans J.-P. Dozon et D. Fassin (dir.), Critique de la santé publique (p. 181-208). Paris Balland.

Fassin, D. (2005). L'altérité de l'épidémie. Les politiques du sida à l'épreuve de l'immigration. Revue européenne des migrations internationales, 17(2), 139-151.

Foucault, M. (1976). Histoire de la sexualité - La volonté de savoir. Paris : Gallimard.

Labelle, M. (2005). Le défi de la diversité au Canada et au Québec. Options politiques, mars, 92-97.

Labelle, M. et Salée, D. (1999). La citoyenneté en question : I'État canadien face à l'immigration et à la diversité nationale et culturelle. Sociologie et sociétés, 31(2), 125-144.

Lazarus, N. (dir.). (2006). Penser le post-colonial. Une introduction critique. Paris : Éditions Amsterdam.

Lorcerie, F. (1997). La catégorisation sociale de l'immigration est-elle coloniale? Hommes et Migrations, 1207, 7886.

Memmi, A. (2005). Portrait du colonisé précédé du portrait du colonisateur. Paris : Gallimard.

Ng, R. (1993). Racism, sexism and nation building in Canada. Dans C. R. McCarthy et W. Crichlow (dir.), Race, Identity and Representation in Education (p. 50-59). New York : Routledge.

Pinell, P. (1992). Naissance d'un fléau. Histoire de la lutte contre le cancer en France (1890-1940). Paris : Métailié.

Roy, B. (2003). Sang sucré, pouvoirs codés, médecine amère. Diabète et processus de construction identitaire: les dimensions socio-politiques du diabètes chez les Innus de Pessant. Québec: Presses de I'Université Laval.

Roy, B. (2004). Vulnérabilité des autochtones ou regard tutélaire des milieux de la santé. In F. Saillant, M. Clément et C. Gaucher (dir.), Identité, vulnérabilité, communautés (p. 189-229). Québec : Nota Bene.

Alterstice - Revue Internationale de la Recherche Interculturelle, vol. $2, n^{\circ} 2$ 
Saillant, F., Chateauneuf, D., Cognet, M. et Charland, M. (2008.). L'accueil paradoxal des réfugiés parrainés par le gouvernement. Une réflexion sur l'intégration et l'exclusion. Dans E. Gagnon, Y. Pelchat et E. Roberson (dir.), Politiques d'intégration, rapports d'exclusion. Action publique et justice sociale (p. 287-302). Québec : Presses de l'Université Laval.

Saillant, F., Cognet, M., Tremblay, M., Roy, G., Kirstic, J., Charland, M. et collab. (2006). L'Autre chez Soi. Lien social, identités et significations des pratiques humanitaires auprès des réfugiés au Québec (Rapport de recherche). Québec: FQRSC.

Schnapper, D. (2007). Qu'est-ce que l'intégration? Paris : Gallimard.

Streiff-Fénart, J. (2002). Modèle républicain et discriminations ethniques : un dilemme français. Faire Savoirs, 1, 6976.

Taguieff, P.-A. (1998). Le racisme. Flammarion

Theureau, J. (2010). Les entretiens d'autoconfrontation et de remise en situation par les traces matérielles et le programme de recherche 'cours d'action'. Revue d'anthropologie des connaissances, 4(2), 287-322.

Todorov, T. (1991). La conquête de l'Amérique. La question de l'autre. Paris : Seuil.

Trudel, M. (2004). Deux siècles d'esclavage au Québec. Montréal : Hurtubise.

Weil, P. (2005). La République et sa diversité. Immigration, intégration, discrimination. Paris : Le Seuil.

Wieviorka, M. (1999). La multiculture, réponse aux menaces philosophiques. Transversales Sciences/Culture, 60. En ligne à : http://multitudes.samizdat.net/La-multiculture-reponse-aux.html

Wiewiorka, M. et Ohana, J. (2001). La différenciation culturelle. Une reformulation des débats. Dans M. Wiewiorka et J. Ohana (dir.), La différence culturelle. Une reformulation des débats (p. 414-427). Paris : Balland.

Winter, E. (2006, 11 août). Between American Gesellschaft and Québécois Gemeinschaft: Constructing the Boundaries of the Canadian Multicultural Nation. Communication présentée au $101^{\mathrm{e}}$ congrès annuel de l'American Sociological Association, Montréal, Canada. 